\title{
Masquerade Syndrome: Diagnostic Challenges in a Case of Primary Central Nervous System Lymphoma
}

\author{
Michael Liam O'Sullivan', Anastasios Catanzaro², Kyle Swerhun², Sanjoy K Gupta ${ }^{3 *}$ \\ ${ }^{1}$ St. Joseph's Family Medical and Dental Centre, London, ON, Canada \\ ${ }^{2}$ Department of Biology, Lakehead University, Thunder Bay, ON, Canada \\ ${ }^{3}$ Associate Professor (Clinical Sciences), Northern Ontario School of Medicine, Lakehead University, Thunder \\ Bay, ON, Canada \\ *Corresponding Author: Sanjoy K Gupta, MD, PhD, FRCSC, Associate Professor (Clinical Sciences), \\ Northern Ontario School of Medicine, Lakehead University, Thunder Bay, ON, Canada; \\ Email: sgupta@tbaytel.net
}

Received Date: 08-09-2021; Accepted Date: 30-09-2021; Published Date: 07-10-2021

Copyright $^{\oplus} 2021$ by Gupta SK, et al. All rights reserved. This is an open access article distributed under the terms of the Creative Commons Attribution License, which permits unrestricted use, distribution and reproduction in any medium, provided the original author and source are credited.

\begin{abstract}
The objective of this investigation was to present a case of masquerade syndrome secondary to primary CNS lymphoma in a Northern Ontario community. Masquerade syndrome of primary CNS lymphoma in the eye can often be misinterpreted as an immune-mediated process, owing to its tendency to first present with only vitreous cell, or chronic uveitis. Correct diagnosis of intraocular primary CNS lymphoma requires clinical suspicion and ophthalmologists should have a low threshold for triggering a diagnostic work-up of older and immunocompromised patients with a characteristic presentation.
\end{abstract}

\section{Keywords}

Masquerade Syndrome; Intraocular Lymphoma; CNS Lymphoma; Vasculitis; Temporal Arteritis 


\section{Introduction}

Primary Central Nervous System Lymphoma (PCNSL) is a malignant neoplasm that can first present in the lymph of the brain, spinal cord and in 1-2\% of extra-nodal cases, intraocularly as Primary Vitreoretinal Lymphoma (PVRL) [1]. CNS lymphoma with ocular involvement is difficult to correctly diagnose, owing to its tendency to masquerade as a benign inflammatory condition. We present a woman with undiagnosed PCNSL masquerading as temporal arteritis causing a Central Retinal Artery Occlusion (CRAO).

\section{Case Report}

A 72-year-old female living in a remote and part of Northern Ontario presented to the Canadian National Institute for the Blind's Medical Mobile Care Unit (CNIB Eye Van) on September 3, 2015 with blurred vision in the left eye: visual acuity 20/20 -1 OD and 20/30-3 OS. She was found to have a $2+$ posterior capsule opacification OS. Yttrium-Aluminum-Garnet (YAG) laser capsulotomy was performed.

She presented again five days later to the CNIB Eye Van with worsening vision loss OS (PH to 20/40) and vitreous haze. She was referred to an ophthalmologist (SKG) in Thunder Bay, $\mathrm{ON}$ in order to rule out a retinal tear and subsequent follow-up.

In November, she presented to clinic with a picture of systemic vasculitis OS. A retinal tear was ruled out and visual acuity was 20/30 OD and 20/40 OS. OCT in the left eye revealed a small Pigment Epithelial Detachment (PED) and overlying Epiretinal Membrane (ERM). Fluorescein angiography showed areas of retinal ischemia, bilaterally. Her erythrocyte sedimentation rate was mildly elevated, carotid doppler showed a non-occlusive plaque in the left common carotid artery and an echocardiogram revealed only mild tricuspid valve regurgitation, but was otherwise normal. A $5 \mathrm{~mm}$ temporal artery biopsy was performed on the left side and was shown to be negative for temporal arteritis. She underwent a second, bilateral temporal artery biopsy which was also shown to be negative for temporal arteritis. Her presentation was nonetheless most in keeping with temporal arteritis and she was given prednisone (50 mg, PO, daily x 1 month) and began a course of methylprednisolone ( $1 \mathrm{~g}, \mathrm{IV}$, daily $\mathrm{x} 3$ days), showing improvement of symptoms.

This patient resided in a remote community in Northern Ontario and access to care was a significant barrier encountered during the course of her treatment. It proved difficult for her to complete the 5-hour journey for follow-up appointments in the winter, which further impeded her care and progression towards a correct diagnosis.

In March 2016, she was found to have a partial thickness macular hole and epiretinal membrane and received an ERM peel procedure and pars plana vitrectomy without complication. In the following 6 months, her visual acuity at regular follow-up appointments wavered between 20/50 and 20/80 OS with significant vitreous haze, always acutely contingent upon steroid

O’Sullivan ML | Volume 2; Issue 3 (2021) | JOAR-2(3)-023 | Case Report

Citation: Gupta SK, et al. Masquerade Syndrome: Diagnostic Challenges in a Case of Primary Central

Nervous System Lymphoma. J Ophthalmol Adv Res. 2021;2(3):1-6.

DOI: http://dx.doi.org/10.46889/JOAR.2021.2304 
administration (prednisolone $1 \%$ gtts OS 1 drop QID). During this time, she also completed a series of three monthly Lucentis injections OS.

In late August 2016, she presented with No Light Perception (NLP) OS. In order to curb her symptoms, she was given two consecutive 1-month courses of prednisone PO $50 \mathrm{mg}$ daily, the second course supplemented with methylprednisolone $1 \mathrm{~g}$ IV x 5 days. Fluorescein angiography showed diffuse areas of hyper fluorescence indicating leakage and areas of hypo fluorescence which indicated retinal ischemia OS > OD. In October 2016, she began to suffer from nocturnal diaphoresis. FA on December 6, 2016 showed advanced ischemia OS and she continued to receive anti-Vascular Endothelial Growth Factor (VEGF) injections (Fig. 1).

In January 2017, she was admitted to her local hospital with breathing difficulties, where a CT angiogram of her thorax showed bilateral pulmonary embolism. In February, 18 months after first presenting to ophthalmology, her condition began to further deteriorate and she was transferred to Thunder Bay Regional Health Sciences Centre. Over the course of two weeks, she suffered progressive weakness beginning in her lower extremities and she regressed from a baseline ambulation using a 2-wheeled walker to bed-bound global weakness, dysphagia and ptosis, only able to talk with great effort. A lumbar puncture was performed on March 6, 2017 which showed a B-cell lymphoma with co-expression of CD5 and CD10, indicating diffuse Bcell lymphoma or Burkitt's lymphoma as the primary CNS lesion. She received two cycles of high-dose methotrexate but her condition deteriorated significantly. She was subsequently transferred back to her community hospital for end-of-life care. She passed away on April 19, 2017. 


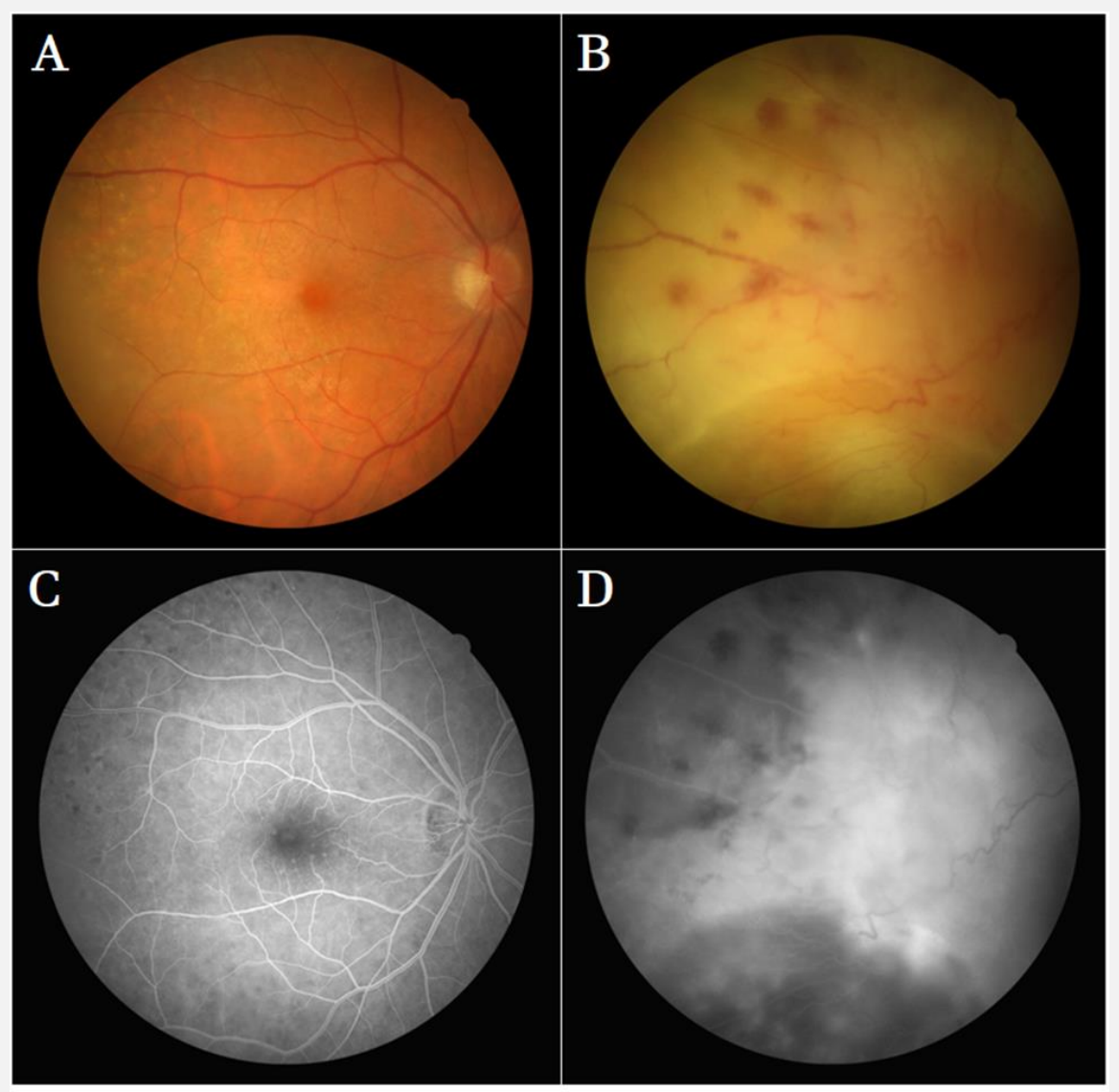

Figure 1: Fundus photographs and fluorescein angiography obtained December 6, 2016 using Canon CX-1 Mydriatic Retinal Camera. (A): OD Fundus photo with yellow, sub-retinal deposits at the periphery of superior temporal arcade, corresponding to punctate hypofluorescence in $\mathrm{C}$; (B): OS fundus photo showing diffuse ischemia with sub-retinal hemorrhage and deposit, venous dilatation, vitreous haze; (C): OD FA showing punctate hypofluorescence characteristic of lymphoma cell deposition; (D): OS FA showing ischemic hypofluorescent areas, large areas of hyperfluorescence (diffuse leakage).

\section{Discussion}

Masquerade syndrome is characterized by intraocular inflammatory cells of a non-immunemediated etiology, merely posing as an immune-mediated process [2]. Primary CNS lymphomas are included among potential true etiologies of masquerade syndrome and are known to have ocular findings in $12-25 \%$ of cases [3]. Ocular manifestations of primary CNS lymphoma typically initiate with only vitreous inflammation, making these cases easy to misinterpret as immune-mediated [4]. Later findings include yellow, sub-retinal deposits secondary to detachment of the Retinal Pigment Epithelium (RPE), considered pathognomonic

O’Sullivan ML | Volume 2; Issue 3 (2021) | JOAR-2(3)-023 | Case Report

Citation: Gupta SK, et al. Masquerade Syndrome: Diagnostic Challenges in a Case of Primary Central Nervous System Lymphoma. J Ophthalmol Adv Res. 2021;2(3):1-6. 
for PVRL [5]. Cases of vitreoretinal lymphoma are described in the literature masquerading as chronic uveitis, retinal artery occlusion, as well as diffuse retinal vasculitis [5-8].

The difficulty involved in making the correct diagnosis is compounded by the fact that visual symptoms associated with ocular lymphoma will typically have a dependent response to steroid treatment in half of presentations and also that the treatment for immune-mediated uveitis (which is oft initiated) will make diagnosis by vitreous biopsy more difficult $[3,5,9]$. The case that we present here fits the model of steroid-dependent relief of visual symptoms. Our report reinforces that inflammatory vision loss symptoms which present as being intimately tied to steroid therapy should be regarded as a harbinger of masquerade syndrome secondary to PVRL or PCNSL, with high suspicion. The gold-standard for diagnosis of PCNSL is contrastenhanced MRI and lumbar puncture, with vitreous biopsy to assess for intraocular involvement [10].

Fluorescein angiography is also helpful in signalling PVRL. Fluorescein angiography performed later in the disease course of our patient showed areas of small punctate hypo fluorescence corresponding to yellow, sub-retinal deposits on fundus photography (Fig. 1). This finding is characteristic of intraocular lymphoma and represents RPE atrophy secondary to deposition of lymphoma cells, usually remaining hypo fluorescent through early to late stages on FA [11,12]. Intraocular lymphoma is also characterized on FA by mottled hyper fluorescence as well as peri-vascular staining, which could explain the hyper fluorescence findings we observed in our patient's left eye [12]. Peri-vascular staining was evident in our patient (Fig. 1).

PCNSL and PVRL are highly fatal diagnoses that require clinical suspicion in order to correctly identify. PVRL has a mean survival of 32 months and PCNSL has a 1-year survival rate of 25$40 \%$ if treated and relapsing [4]. The case that we report here considers the importance of having a low threshold for suspicion of neoplastic masquerade syndrome and subsequent investigation with MRI, lumbar puncture and vitreous biopsy when older patients present with vision loss associated with yellow sub-retinal plaques or vitreous haze, particularly if the patient is immunocompromised.

\section{Conclusion}

Masquerade syndrome of primary CNS lymphoma in the eye can often be misinterpreted as an immune-mediated process, owing to its tendency to first present with only vitreous cell, or chronic uveitis. We present a woman with undiagnosed PCNSL masquerading as temporal arteritis causing a Central Retinal Artery Occlusion (CRAO). Ophthalmologists should have a low threshold for triggering a diagnostic work-up of older and immunocompromised patients with a characteristic presentation. 


\section{References}

1. Freeman LN, Schachat AP, Knox DL, Michels RG, Green WR. Clinical features, laboratory investigations and survival in ocular reticulum cell sarcoma. Ophthalmol. 1987;94(12):1631-9.

2. Tsai T, O'Brien JM. Masquerade syndromes: malignancies mimicking inflammation in the eye. Int Ophthalmol Clin. 2002;42(1):115-31.

3. Read RW, Zamir E, Rao NA. Neoplastic masquerade syndromes. Surv Ophthalmol. 2002;47(2):81-124.

4. Chan CC, Sen HN. Current concepts in diagnosing and managing primary vitreoretinal (intraocular) lymphoma. Discovery Med. 2013;15(81):93.

5. Cimino L, Coassin M, Chan CC, Marchi S, Belpoliti M, Fanti A, et al. Vitreoretinal lymphomas misdiagnosed as uveitis: Lessons learned from a case series. Indian J Ophthalmol. 2016;64(5):369.

6. Zhang P, Tian J, Gao L. Intraocular lymphoma masquerading as recurrent iridocyclitis: findings based on in vivo confocal microscopy. Ocular Immunol Inflammation. 2018;26(3):362-4.

7. Gass JD, Trattler HL. Retinal artery obstruction and atheromas associated with non-Hodgkin's large cell lymphoma (reticulum cell sarcoma). Arch Ophthalmol. 1991;109(8):1134-9.

8. Katoch D, Bansal R, Nijhawan R, Gupta A. Primary intraocular central nervous system lymphoma masquerading as diffuse retinal vasculitis. Case Rep. 2013;2013:bcr2013009354.

9. Hormigo A, Abrey L, Heinemann MH, DeAngelis LM. Ocular presentation of primary central nervous system lymphoma: diagnosis and treatment. BR J Haematol. 2004;126(2):202-8.

10. Sen HN, Bodaghi B, Hoang PL, Nussenblatt R. Primary intraocular lymphoma: diagnosis and differential diagnosis. Ocular Immunol Inflammation. 2009;17(3):133-41.

11. Davis JL. Intraocular lymphoma: a clinical perspective. Eye. 2013;27(2):153-62.

12. Fardeau C, Lee CP, Merle-Béral H, Cassoux N, Bodaghi B, Davi F, et al. Retinal fluorescein, indocyanine green angiography and optic coherence tomography in non-Hodgkin primary intraocular lymphoma. Am $\mathrm{J}$ Ophthalmol. 2009;147(5):886-94. 